This is a post-print version of the following article: Ezpeleta, L., Penelo, E., Navarro, J.B., de la Osa, N., \& Trepat, E. (2020). Transdiagnostic trajectories of irritability and oppositional, depression and anxiety problems from preschool to early adolescence. Behaviour Research and Therapy, 134. https://doi.org/10.1016/j.brat.2020.103727

Transdiagnostic trajectories of irritability and oppositional, depression and anxiety problems

from preschool to early adolescence

$$
\begin{aligned}
& \text { Lourdes Ezpeleta }{ }^{1,2} \\
& \text { Eva Penelo }{ }^{1,3} \\
& \text { J. Blas Navarro }{ }^{1,3} \\
& \text { Núria de la Osa }{ }^{1,2} \\
& \text { Esther Trepat }{ }^{1,2} \\
& \text { Universitat Autònoma de Barcelona } \\
& \text { 08193 Bellaterra (Barcelona). SPAIN } \\
& { }^{1} \text { Unitat d'Epidemiologia i de Diagnòstic en Psicopatologia del Desenvolupament } \\
& \text { Universitat Autònoma de Barcelona } \\
& \text { 08193 Bellaterra (Barcelona). SPAIN } \\
& \text { Universitat Autònoma de Barcelona } \\
& \text { 08193 Bellaterra (Barcelona). SPAIN }
\end{aligned}
$$

Mailing address:

Lourdes Ezpeleta

Departament de Psicologia Clínica i de la Salut. Edifici B

Universitat Autònoma de Barcelona

08193 Bellaterra (Barcelona). SPAIN

Phone: (34) 935812 883; $\quad$ E-mail: Lourdes.ezpeleta@uab.cat 


\begin{abstract}
Irritability is a transdiagnostic symptom in oppositional defiant disorder, depression, and anxiety, all highly comorbid disorders but with unknown simultaneous evolution. The aim was to obtain the developmental trajectories of simultaneous irritability and oppositional, depression, and anxiety problems from preschool age to early adolescence. A sample of 493 community children was followed up annually from ages 3 to 11 years and assessed using categorical and dimensional measures answered by parents and teachers. Latent Class Growth Analysis for four parallel processes was used to identify distinct groups of individual trajectories for irritability and oppositional, depression, and anxiety problems. Outcomes at ages 11 and 12 were compared among trajectories using regression models and multiple comparisons. A 3-class model showed the highest entropy (.961) and adequate posterior probabilities of class membership ( $\geq .969)$. Class 1 ( $n=331,67.1 \%)$ was made up of children with stable low scores in all the variables; class $2(n=55,11.2 \%)$ of children with high depression, anxiety, and irritability and above the mean stable profiles for oppositional problems; and class 3 ( $n=107,21.7 \%)$ of children with medium-high increasing irritability and oppositional problems and on the mean depression and anxiety. The classes with symptomatology and irritability (2 and 3) clearly differed from class 1 (low) at baseline and in outcomes. The course of irritability and oppositional, depression, and anxiety problems from ages 3 to 11 years differed qualitatively and quantitatively across subgroups of children. The 3 classes identified may help to guide clinicians’ decision-making regarding treating irritability and its comorbid disorders.
\end{abstract}

Keywords: Anxiety, Depression, Developmental trajectories, Irritability, Oppositional defiant disorder, Transdiagnostic. 
Trajectories of irritability, oppositionism, depression and anxiety 3

\section{Transdiagnostic trajectories of irritability and oppositional, depression, and anxiety problems from preschool to early adolescence}

Comorbidity between internalizing and externalizing disorders in childhood is very prevalent, has a high continuity, and is associated with worst outcomes (Willner, GatzkeKopp, \& Bray, 2016). The relationship between the two kinds of problems has been explained from different perspectives, such as that one type of disorder leads to the other (externalizing problems due to failure experiences increase the risk of internalizing problems, or internalizing problems make it difficult to self-regulate, increasing the risk of externalizing problems), that they share common risk factors, and that both have a bidirectional effect (Angold, Costello, \& Erkanli, 1999; Oh, Greenberg, Willoughby, \& Family Life Project, 2020). Based on the high comorbidity observed, it has been suggested that psychopathology models that consider the co-occurrence of internalizing and externalizing symptoms may be more informative to prevent adverse long-term outcomes than those focused only on specific symptoms (Sallis et al., 2019). In this line, interventions should focus on the co-occurrence of symptoms to prevent long-term impacts (Sallis et al., 2019).

Three disorders on the externalizing and internalizing spectrums that tend to frequently co-occur in childhood are oppositional defiant disorder (ODD), depression (DEP), and anxiety (ANX) (Lavigne, Gouze, Bryant, \& Hopkins, 2014; Wakschlag et al., 2015). Irritability is a transdiagnostic symptom in all three (Toohey \& DiGiuseppe, 2017): it is one of the dimensions that define ODD (Stringaris \& Goodman, 2009); the DSM-5 considers that depressed mood in children may be manifested as irritable mood; and the definition of some anxiety disorders also includes irritability (i.e. generalized anxiety disorder). In a metaanalysis, Vidal-Ribas, Brotman, Valdivieso, Leibenluft, and Stringaris (2016) reported that irritability is associated with odds ratios of 1.8 for future depression, 1.7 for anxiety problems, 
and 2.6 for ODD. From early ages, irritability has a direct effect on later depression, anxiety, and oppositional problems (Ezpeleta, Penelo, de la Osa, Navarro, \& Trepat, 2019; Wakschlag et al., 2015). Above and beyond DSM symptoms, irritability from early ages shows incremental value for later internalizing, disruptive behavior disorders, and impairment (Wakschlag et al., 2018).

Irritability is defined as an excessive reactivity to negative emotional stimuli (Leibenluft \& Stoddard, 2013) characterized by the emotion of anger, and temper outbursts as the behavioral manifestation (Stringaris, 2011). Irritable children are touchy or easily annoyed, have a low threshold for experiencing anger in response to frustration, and present anger/temper outbursts. According to Wakschlag et al.'s (2018; 2019) heuristic for specifying the development of irritability, it is a normative expression when frustrated, which the child learns to control as the ability to regulate emotion develops. Attainment of competence in emotion self-regulation is a milestone to protect against mental health problems throughout life. During the preschool period, the growth of the cortical structures mediating anger facilitates executive functions that help to self-regulate anger (such as cognitive flexibility) and to execute adaptive responses. The context, frequency, persistence, intensity, and low threshold of elicitation define abnormality, which may involve a dysfunction in reward (hypersensitivity) and threat (approach) processing, abnormal subcortical activation, and altered prefrontal functions (the abnormal activation of the dorsolateral prefrontal cortex during frustration). Wakschlag et al. (2019) highlight that persistent patterns of early irritability exponentially increase the risk of developing mental health problems such as mood and behavioral problems, and functional impairment.

Irritability is, therefore, a key component for explaining developmental pathways within and between internalizing and externalizing disorders into adulthood (Burke et al., 2014) and there is a need to study the transdiagnostic importance of its early emergence 
(Wakschlag et al., 2018). Early detection becomes a priority and to this end within-person strategies that account for developmental change over time using repeated measures may be of help to warrant enough stable patterns for probabilistic risk estimates (Wakschlag et al., 2019). Person-centred approaches are suitable strategies to meet this challenge. Personcentred analyses enable groups of individuals with similar responses in relation to the studied variables to be found. Latent Class Growth Analysis (LCGA) helps to identify groups of similar children (classes) according to their longitudinal trajectories. LCGA with parallel processes identifies groups of children based on the simultaneous growth of the variables under study. This approach was selected here since the focus was the simultaneous codevelopment of comorbid and transdiagnostic symptomatology. In the present study, latent classes of trajectories with different growth parameters of irritability and oppositional, depression, and anxiety problems were considered simultaneously.

Information about univariate trajectories of irritability (Ezpeleta, Granero, de la Osa, Trepat, \& Domènech, 2016; Pagliaccio, Pine, Barch, Luby, \& Leibenluft, 2018; Wiggins, Mitchell, Stringaris, \& Leibenluft, 2014), and oppositional (Althoff, Kuny-Slock, Verhulst, Hudziak, \& van der Ende, 2014), depression, and anxiety problems (de Lijster et al., 2019) is available, but few previous works have studied any combination of them using parallel processes that show their simultaneous development. In one such study, Leadbeater, Thompson, and Gruppuso (2012) studied the trajectories of the co-occurrence of anxiety, depression, and oppositional symptoms from adolescence until the transition into adulthood (12 to 26 years) in a community sample. The results showed that the anxiety, depression, and oppositional levels co-occurred at each time point and, except for anxiety and oppositional problems, they also co-develop over time. Adolescents that started high in one domain were also consistently high in the others at each assessment point, and by adulthood anxiety symptoms (females) and depression symptoms (males) were still increasing, whereas 
oppositionality had declined or stabilized. Fanti and Henrich (2010) studied the development of co-occurring internalizing and externalizing symptoms in a community sample over a 10year period from ages 2 to 12 years. Distinct classes representing different co-occurring evolutions of the symptoms (pure continuous externalizing, pure internalizing, highinternalizing and high-desisting externalizing, co-occurring problems) were identified. The children in the chronic co-occurring externalizing and internalizing problems class differed from the children in the other classes in that they had more peer problems and were more rejected, suggesting a lack of social skills. In a community sample of 3- to 10-year-old children, Ip, Jester, Sameroff, and Olson (2019) also obtained four classes of co-occurring internalizing and externalizing symptoms: low (21\%), low-moderate (55\%), rising (15\%), and severe-decreasing (9\%). Being male and low executive control were risk factors of pertaining to the rising class in comparison with low profiles. These studies have shown that few children develop “pure” disorders, have identified homogenous classes of children with similar patterns of symptomatology, and suggest that an approach that includes their codevelopment may be more informative to better understand comorbid disorders.

The co-development of irritability considering the presence of concurrent oppositional, depression, and anxiety problems is unknown. Knowledge of the specific evolution of irritability and its comorbid disorders in different classes would help to be better informed about their developmental psychopathology, which would be useful to develop prevention and intervention strategies based on the developmental phenomenology observed, and to refine the treatments for each class. The goal was to identify models that represent the concurrent evolution of irritability with oppositional, depression, and anxiety symptoms from preschool age to early adolescence (ages 3 to 11 years), including homogeneous groups of children with this symptomatology who may have similar clinical needs. Given the transdiagnostic nature of irritability, it was expected to co-develop close in proximity to the other symptoms and that 
there would be a class of comorbid symptomatology across development; in other words, children in trajectories of high scores on all the measures with similar shapes and slopes throughout development would be found. Given the inclusion of the irritability dimension in ODD, a specific class clustering oppositional problems and irritability with marginal depression and anxiety symptoms was also expected to be found. Since homotypic comorbidity is higher than heterotypic comorbidity (Angold et al., 1999), a class with similar development of depression, anxiety, and irritability symptoms and marginal oppositional problems was also expected to emerge. An additional goal was to study the baseline variables and outcomes of each trajectory to know their developmental psychopathology, thus informing prevention and intervention programs. It was hypothesized that classes with elevated irritability and symptoms would show worse clinical characteristics than the others. This is the first study to analyze the co-development of this symptomatology including boys and girls and four parallel processes (irritability and oppositional, depression, and anxiety problems) with LCGA.

\section{Method}

\section{Participants}

The sample comes from a longitudinal study of behavioral problems starting at age 3 years described in Ezpeleta, de la Osa, and Doménech (2014). A two-phase design was employed to obtain a representative sample of the target population. In the first phase of sampling, an initial group of 2,283 children was randomly selected from the census of infant schools in Barcelona (Spain), out of which 1,341 families (58.7\%) agreed to participate (33.6\% high socioeconomic status (SES), 43.1\% middle SES, and 23.3\% low SES; 50.9\% boys). To ensure the participation of children with possible behavioral problems, the parentrated Strengths and Difficulties Questionnaire (SDQ) conduct problems scale (Goodman, 
2001) plus four ODD DSM-IV-TR symptoms not included in the SDQ questions was used for screening. Two groups were potentially considered: the first screen-positive, which included all the children with SDQ scores $\geq 4$, in percentile 90 , or with a positive response to any of the 8 DSM-IV ODD symptoms ( $N=417 ; 49.0 \%$ boys); and the second screen-negative, a random group comprising the $28 \%$ of children who did not reach the positive threshold $(N=205$; $51.2 \%$ boys). Refusals in this phase $(n=135 ; 10.6 \%)$ did not differ in terms of sex $(p=.815)$ or type of school (state or semi-private) $(p=.850)$ from the children who agreed to participate (the only difference was in SES, with a higher participation percentage for high socioeconomic levels, $86.2 \%$ vs. $73.6 \% ; p=.007)$.

The sample for the follow-up (the second phase of the sampling design) included 622 children (mean age $=3.77$ years; $S D=0.33 ; 96.9 \%$ born in Spain) who were followed up yearly from age 3 to 12 years. The data from age 3 to 11 years ( 9 assessment points) were used to estimate the trajectories. Information was available from four waves or less for 129 children (20.7\%), from five waves for 41children (6.6\%), from six waves for 47 children (7.6\%), from seven waves for 57 (9.2\%), from eight waves for 109 (17.5\%), and from all nine waves for 239 children (38.4\%). It was decided to exclude the children with data for less than half of the 9 waves ( 4 or less waves), leaving a sample of 493 children (66.5\% screenpositive; 249 (50.5\%) girls; 91.7\% Caucasian; 34.1\% high SES, 46.6\% middle-high or middle SES, and 19.3\% middle-low or low SES, according to Hollingshead's (1975) index). The data from 5 or more of the 9 possible annual follow-ups between ages 3 and 11 years thus represented $79.3 \%$ of the sample of 622 children included in the follow-up (78.8\% from the positive screening group and $80.1 \%$ from the negative screening group; $\chi^{2}(1)=0.13, p=$ .717). The mean age (and $S D$ ) at each follow-up point was as follows: 3.76 (0.34) for followup 1, 4.67 (0.35) for follow-up 2, 5.70 (0.36) for follow-up 3, 6.60 (0.35) for follow-up 4, 
7.69 (0.35) for follow-up 5, 8.65 (0.35) for follow-up 6, 9.66 (0.36) for follow-up 7, 10.60 (0.33) for follow-up 8, and 11.60 (0.35) for follow-up 9.

\section{Instruments}

\section{Trajectories}

Irritability. The focus of the study was irritability defined with the symptoms 'touchyeasily annoyed', 'angry and resentful', and 'loses temper' (Ezpeleta, Granero, de la Osa, Penelo, \& Domènech, 2012). Accordingly irritability was measured with 3 items using a 3point ordered response format ranging from 0 (not true) to 2 (somewhat true). Specifically, the symptom "loses temper" was measured with one of the items of the conduct problems scale (i.e. temper tantrums) from the Strengths and Difficulties Questionnaire (SDQ) (Goodman, 1997). The symptoms touchy-easily annoyed' and 'angry and resentful' were measured with two additional items (i.e. touchy, angry-resentful) that were further included in the SDQ as part of the assessment protocol in the original design of the current study. The parents reported these symptoms each year ( $M d$ of ordinal alpha values over the 9 follow-ups: $\left.\alpha_{0}=.82\right)$. T-scores were used.

The Child Behavior Checklist/1느-5 and 6-18 (CBCL; Achenbach \& Rescorla, 2000; 2001) are questionnaires answered by parents which evaluate behavioral and emotional problems rated on a scale from 0 (not true) to 2 (very or often true). The focus in this study was on the DSM5-Oriented scales: oppositional problems, depressive problems, and anxiety problems. The medians of ordinal $\alpha$ for the 9 follow-ups were $.85, .83$ and .85, respectively. T-scores were used.

Baseline variables (age 3) 
The Children's Behavior Questionnaire Short Form (Putnam \& Rothbart, 2006) measures reactive and self-regulative temperament (94 items on a 7-point Likert-type scale) reported by parents. The dimensions negative affectivity, effortful control, and surgency were considered. Cronbach’s alphas were $.83, .79$, and .82, respectively.

The Alabama Parenting Questionnaire-Preschool (Frick, 1991; de la Osa, Granero, Penelo, Doménech, \& Ezpeleta, 2014) measures parental practices in 42 items (1: Never to 5: Always) that form three dimensions: positive discipline, inconsistent parenting, and punitive parenting. Given that many of the items, mainly in the last two dimensions, showed high skewness and low variability, the ordinal alpha was calculated, giving values of .85, .70, and .63 , respectively.

The Adult Self-Report (ASR; Achenbach \& Rescorla, 2003) assesses dimensional psychopathology reported by mothers. It contains 126 items (0: not true to 2: very true/often true). Ordinal alpha ranged from .75 (thought problems) to .96 (total), except for rulebreaking (.66).

\section{Outcomes}

The Diagnostic Interview for Children and Adolescents for Parents of Preschool and Young Children (DICA-PPYC; Ezpeleta, de la Osa, Granero, Domènech, \& Reich, 2011) is a computerized, semi-structured diagnostic interview for assessing the most common psychological disorders from age 3, according to the DSM-5 criteria. Attention deficithyperactivity disorder (ADHD), ODD, and anxiety disorders (including separation anxiety, generalized anxiety, specific phobia, and social phobia) were included as outcomes at age 11 . There were not enough cases of conduct disorder or major depression to be included in the study. After assessing the symptoms of each disorder, the interviewer asked about any 
consultations and treatment received. The resultant number of diagnoses from the interview was considered as an indicator of comorbidity.

The Strengths and Difficulties Questionnaire (SDQ; Goodman, 1997), answered by the parents and teachers of 12-year-old children, measures psychopathology dimensionally. The SDQ has 25 items with 3 response options (0: "not true”; 1: "somewhat true”; 2: “certainly true”) organized in 5 scales (emotion, conduct, hyperactivity, peer, and prosocial) and a total score is obtained. Reverse items were coded in the direction of higher scores indicating more psychopathology. Medians of ordinal alpha for the five subscale scores were .80 for parents' ratings ( $\alpha_{0}=.87$ for the total score) and .89 for teachers' ratings $\left(\alpha_{0}=.94\right.$ for the total score).

The Children’s Global Assessment Scale (CGAS; Shaffer et al., 1983; Ezpeleta et al., 1999) is a global measure of functional impairment, which was evaluated at age 12 . Scale scores range from one (maximum impairment) to 100 (normal functioning). Scores above 70 indicate normal adaptation.

\section{Procedure}

The project was approved by the Ethics Committee on Animal and Human Experimentation of the author's institution. The families were recruited at the schools and they gave written consent for the assessment. All the families of the 3-year-old children from participating schools were invited to answer the screening questionnaire. The families who agreed and met the screening criteria were contacted by telephone and interviewed at the school for each annual assessment. The interviewer team was specifically trained and all the interviewers were blind to the screening group. The teachers answered the questionnaires after permission from the families was obtained. 


\section{Data Analysis}

Given the multistage sampling procedure used, all the analyses were weighted by the inverse probability of selection in the second phase of sampling. MPlus 8.3 LCGA for parallel processes was used to identify distinct groups of individual trajectories for irritability (SDQ) and oppositional, depression, and anxiety problem (all three CBCL) T-scores jointly. The Robust Maximum Likelihood (MLR) method of estimation was used, enabling the inclusion of non-normal and incomplete data using the expectation maximization algorithm for missing data with robust standard errors (i.e., full information method). The growth models for SDQ and CBCL scores considered intercept (I) and slope (S; i.e. linear trends) over the nine annual assessments from ages 3 to 11 years, with equal spacing between measurement occasions. The time was rescaled from 3-11 years to 0-8 years so that the first-year assessment (at age 3 years) represented the intercept.

Models with one to six latent classes of growth patterns were obtained. The following criteria were used to determine the best model and to show the best clinical interpretability: larger decrement in AIC and sample-size adjusted BIC (aBIC), greater power and more accurate classification by average posterior probabilities, entropy values equal to or greater than .70, and more than 25 (5\%) participants in a class.

The baseline variables at age 3 and outcomes at age 11/12 were compared among trajectories using linear or logistic regression models with Stata 16, with trajectory as the categorical independent term and outcome as the binary or continuous dependent term. The analysis of each outcome was adjusted by baseline using the same outcome measure obtained at age 3. Multiple comparisons between trajectories were made for the overall statistically significant results, and the Holm-Bonferroni (Holm, 1979) correction applied. Effect sizes were calculated as odds ratios $(O R)$ for binary outcomes and Cohen's $d$ for continuous 
outcomes. In line with (Cohen, 1992), absolute values of $d$ were interpreted as small effect for values 0.20-0.50, medium effect for values 0.50-0.80, and large effect for values $>0.80$.

\section{Results}

Trajectories of irritability, oppositional, depression, and anxiety problems

Table 1 shows the goodness-of-fit indices for the LCGA models from one to six classes. Based on clinical interpretability and the second largest decrement in AIC and aBIC, the 3-class model (Figures 1a to 1d), which showed the highest entropy (.961) and very high posterior probabilities of class membership ( $\geq$.969), was selected. The quadratic trend of the trajectories was estimated. There were no relevant differences in the estimated parameters between models, including linear and quadratic trends, for any of the trajectories. Therefore, following the parsimony principle, the linear model approach was selected.

Class 1 ( $n=331,67.1 \%$; Figure 2a) was made up of children with low scores and a stable profile for irritability and oppositional, depression, and anxiety problems (low class); Class 2 ( $n=55,11.2 \%$; Figure 2b) of children with initial high stable depression, anxiety, and irritability, and above the mean stable profiles for oppositional problems (high comorbid class); and class 3 ( $n=107,21.7 \%$; Figure 2c) of children with above the mean increasing irritability, above the mean stable oppositional problems, medium increasing anxiety, and on the mean depression (oppositional-irritability class). Supplementary Table 1 shows the parameter estimates for the final 3-class model.

Table 2 presents the descriptive of the three classes. There were no significant differences among them in the distribution of sex and SES. At baseline, class 1 (low) had lower scores than class 2 (comorbid) and class 3 (oppositional-irritability) in all the variables, except for depression problems in comparison with class 3. Class 2 (comorbid) differed from class 3 (oppositional-irritability) with higher scores for depression and anxiety problems. 
Comparison between class 1 (low) and class 2 (high comorbid depression-anxiety-irritability and oppositional) at baseline and in outcomes

Table 3 shows the baseline variables (age 3) and outcomes (at ages 11 and 12) of the different classes and the comparison among them. In comparison with class 1 (low), the class 2 children (high comorbid) showed higher negative affect, inconsistent parenting, and higher mother's psychopathology at baseline. At the end of the trajectory (age 11), they evidenced a higher risk of presenting a DSM-5 diagnosis of ADHD, ODD, any anxiety, and comorbidity (higher mean number of disorders), and they were more likely to have sought professional help and received treatment for their psychological problems. Their dimensional outcomes were also more adverse, and they showed higher symptomatology in all the SDQ scales (except prosocial) as reported by parents, higher scores in the SDQ scales emotion, peer, prosocial, and total as reported by the teachers, and lower functioning. Effects sizes were in the high range, with Cohen's $d$ around 0.8 or higher (the exception was the difference in inconsistent parenting and prosocial as reported by teacher), and $O R$ higher than 5 .

Comparison between class 1 (low) and class 3 (oppositional-irritability) at baseline and in outcomes

Following Table 3, in comparison with class 1 (low), the children in class 3 (oppositional-irritability) showed higher negative affect, inconsistent parenting at baseline, a higher risk of presenting a DSM-5 diagnosis of ODD, any anxiety, and comorbidity (higher mean number of disorders) at the end of the trajectory (age 11), and a greater likelihood of having sought professional help and received treatment for their psychological problems. Parents' SDQ scores (except prosocial) were highest for the children in class 3, with effect sizes in the moderate to high range ( $d$ between 0.50 and 1.22; with the exception of a low 
effect for negative affect), as were teachers' SDQ emotion and total scores (low effect sizes; $d$ between 0.32 and 0.37 ), and OR between 2.87 and 10.93 .

Comparison between class 3 (oppositional-irritability) and class 2 (high comorbid depression-anxiety- irritability and oppositional) at baseline and in outcomes

In comparison with class 3, class 2 showed higher mother’s psychopathology at baseline and, at the end of the trajectory (age 11), higher comorbidity and higher SDQ teachers' scores for emotion and total (effect sizes for comorbidity between low and moderate to high). Class 3 presented higher scores at baseline for punitive parenting (low effect size).

\section{Discussion}

The high comorbidity between ODD, depression, and anxiety and the fact that these disorders share irritability as a transdiagnostic symptom support the approach of studying the co-occurrence of their joint development. The objective was to discover the empirical classes that represent the concurrent evolution of irritability and oppositional, depression, and anxiety symptoms from ages 3 to 11 and to study their associated clinical variables. The course of irritability and oppositional, depression, and anxiety problems from age 3 to 11 differed across subgroups of children. Three groups were found: a class with low scores through development, a class with high and stable symptoms of depression, anxiety, and irritability plus sustained oppositional symptoms above the mean (comorbid), and a class with sustained above the mean irritability and oppositional and mean anxiety and depression. Class membership informs about levels relevant to the course of irritability and oppositional, depression, and anxiety problems considered simultaneously and may point towards similar clinical needs. The simultaneous modelling of the variables adds value by empirically reflecting the co-occurrence of multiple psychopathological symptomatologies and their 
transdiagnostic symptoms, helping to qualitatively identify different groups of children that require specific and varying interventions. The classes with irritability and psychopathological symptomatology with values above the mean differed on baseline measures and had similar negative outcomes in comparison with trajectories with low values.

The oppositional-irritability classes did emerge and the set hypotheses were partially verified. As expected, irritability co-develops with the rest of the symptomatology. In the classes with symptomatology (classes 2 and 3), the shape and slope of irritability through development was sustained above the mean from the beginning, increasing slightly until preadolescence, from when it is expected to continue or be maintained as puberty advances and with the action of hormones (Oldehinkel, Verhulst, \& Ormel, 2011). This consolidated increase or stable shapes in the development of irritability coexisting with other symptoms highlights the need for early detection and specific interventions, irrespective of what the main associated disorder is, and is further relevant considering that research has shown that even at levels of irritability typically defined as within the normal range there is a risk of bad outcomes, including disruptive behaviour disorders, depression, and anxiety (Wakschlag et al., 2015).

The development of irritability seems to be more overlapped with oppositional problems (Class 3) than with depression and anxiety (Class 2). As was hypothesized, a class with both patterns emerged. A main manifestation of ODD is anger-irritability, defining the disorder (Stringaris \& Goodman, 2009) and now included in DSM-5. About 65\% of the children with ODD presented with high-persistent or increasing irritability, which was associated with poor longitudinal outcomes (disruptive behaviour disorders, functional impairment, internalizing and externalizing problems, and lower anger control) (Ezpeleta et al., 2016). The structure of ODD is currently under discussion and different alternatives have been proposed (Burke, Hipwell, \& Loeber, 2010; Krieger et al., 2013; Rowe, Costello, 
Angold, Copeland, \& Maughan, 2010). However, Burke et al. (2014), who used a wide sample (>16,000 5 to 18 years old) to study the structure of ODD, reported that irritability was not sufficiently distinct from ODD to be considered as a different diagnosis, choosing a bifactor model that included irritability and oppositional behaviour plus a general ODD factor as the best structure of ODD. This close relationship is also manifested developmentally, as was captured in class 3. Interestingly, irritability slightly surpassed the trajectory of oppositional problems from 8 years old onwards, which likely indicates increased gonadal steroid hormone secretion, possibly favouring increased irritability and a predominance of this symptomatology (Oldehinkel, Verhulst, \& Ormel, 2011). This could point to the need to focus on detecting and treating irritability symptoms, especially from age 8 years onwards and, given its ascending slope, to prevent it from preschool age.

Class 2 represents the most severe symptomatology and was the least prevalent. The levels of depression, anxiety, and irritability were the highest and oppositional symptomatology was also high (almost as high as in class 3). As would be expected, the risks and outcomes of this class were very negative (see below) according to both parent and teacher information. Irritability is a transdiagnostic risk marker of decrements in selfregulation (Smith et al., 2019), which can affect different emotions and is explained as an aberrant response to frustrative non-reward and threat (Brotman, Kircanski, Stringaris, Pine, \& Leibenluft, 2017). Irritable children have difficulty learning when to anticipate rewards or punishments and show dysfunctional adaptation when a goal is not attained, and they may also have increased orientation toward threatening stimuli (Stringaris, Vidal-Ribas, Brotman, \& Leibenluft, 2018). The neurobiological basis of these dysfunctions are decreased striatal activity and decreased activation in frontal areas when rewards are omitted, with difficulties modulating amygdala responses (Stringaris et al., 2018). Like irritability, an altered reward system and difficulties in emotion recognition are also critical in the development of 
depression, anxiety, and ODD (Forbes \& Dahl, 2012; Matthys, Vanderschuren, \& Schutter, 2013; Silk, Davis, McMakin, Dahl, \& Forbes, 2012).The identification of this class with the increasingly sustained co-occurrence of depression, anxiety, irritability, and oppositional symptoms from preschool age to early adolescence, which affected about $11 \%$ of the children in the sample, is of remarkable clinical value and places the onus on the field of prevention.

It was expected that a class would be found that included the homotypic comorbidity of depression and anxiety, where irritability would co-develop with marginal scores in oppositional problems. Such a class was not found because oppositional symptoms were also high in class 2, which could indicate that although depression and anxiety are closer than depression or anxiety and oppositional, they still co-develop with a common nexus of irritability. In other words, irritability must be considered to better understand the comorbidity between depression, anxiety, and oppositional problems (Stringaris, 2011).

The classes with symptomatology and irritability (2 and 3) clearly differed from class 1 (low) at baseline and in outcomes. The differences at baseline enabled the identification of varying risks, including temperament, parenting practices, and maternal psychopathology. High negative affect was a marker of pertaining to classes 2 and 3. Note, for instance, the relevance and specificity of the mother's psychopathology in class 2 and punitive parenting in class 3. Any type of maternal psychopathology, a well-recognized risk factor of child psychopathology (Goodman et al., 2011; Sellers et al., 2013) was specifically associated with the most severe comorbid trajectory 2. In the same line, punitive parenting, typically associated to oppositional defiant disorder through coercion cycle (Patterson, 2002) was specifically associated to trajectory 3. Pertaining to these classes also increased the odds of presenting ODD or any anxiety disorder at age 11 by between 4 - and almost 11 -fold. The children in these classes had a higher risk of having received professional care and treatment and, according to parents and teachers, they had more severe symptomatology on dimensional 
measures. The ORs and the effect sizes for the comparisons were relevant, especially for the comparison between classes 1 and 2. It is important to note that, with few exceptions, classes 2 and 3 had similar bad outcomes, meaning that the co-development of irritability and its associated internalizing or externalizing symptomatology leads to a bad prognosis. Symptomatology in class 2 (comorbid) was clearly more severe but few differences in the outcomes emerged in comparison with class 3 (oppositional-irritability): class 2 led to a higher number of DSM-5 disorders, and teachers identified more severe symptomatology.

This is the first study to simultaneously analyze the co-development of irritability and oppositional, depression, and anxiety symptoms from preschool age to early adolescence in a 9 yearly based follow-up of a wide sample of boys and girls from the general population using LCGA parallel processes. Independent outcomes at ages 11 and 12 were also studied.

Trajectories were based on parents' information and several tools and informants were used to recruit information on outcomes. However, the results should be interpreted considering some limitations. The sample was a community sample in which, as expected, there was a low rate of psychopathology, meaning that outcomes such as conduct disorder or major depression could not be estimated. The data was modelled through LCGA rather than through Growth Mixture Modelling (GMM), which allows variation across individuals within classes, given that in our case GMM did not converge, as often happens (Wickrama, Lee, Walker-O'Neal, \& Lorenz, 2016). Despite LCGA being less realistic than GMM, it can be considered to be a nested and more parsimonious model of GMM. Furthermore, the three-classes model with linear trend did not differ remarkably if the quadratic trend was added. The results about parenting practices should also be interpreted with caution given that the two scales of the APQ questionnaire had low values of internal consistency (inconsistent and punitive parenting). Finally, our trajectories arrived at age 11, and it is possible that trajectories of 
irritability and depression/anxiety might differ in later stages of development, when these disorders have their onset and first peak (Chahal, Gotlib, \& Guyer, 2020).

The classes identified may help to guide clinicians’ decision-making regarding treating irritability and its comorbid disorders. High comorbidity among disorders has fostered the development of transdiagnostic treatments, potentially improving cost-effectiveness and efficiency (Chu, Temkin, \& Toffey, 2016). There are several approaches to developing transdiagnostic treatments: a) the core dysfunction approach, which targets common underlying dysfunction among disorders; b) the common elements approach, which focuses on the common therapeutic procedures used for the disorders; and c) the principle guide approach, which focuses on the core principles of therapeutic change in the disorders (Marchette \& Weisz, 2017). Along these lines, the results obtained in this study may inform core dysfunction approach treatments, which would target irritability as a common underlying dysfunction among oppositional, depression and anxiety problems. According to our results, these programs should foresee a group of children with irritability and oppositionality and another group with irritability and comorbid symptoms of depression, anxiety, and oppositionality. Treatment for the first group should be based on parental training in behavior modification and self-control techniques for children focused mainly on anger control, which is the treatment of choice for children with ODD. For the second group, emotions should be approached through a wider spectrum of strategies, given the high comorbidity. A protocol such as the Unified Protocol for Transdiagnostic Treatment of Emotional Disorders in Children (Ehrenreich, Goldstein, Wright, \& Barlow, 2009), which targets high negative emotion, emotional reactivity, and emotion regulation deficits common across emotional disorders, may be indicated for this group. This protocol has been tested in children with depression and anxiety and also has proved promising initial results in a small sample of 
children with disruptive behavior disorders (Hawks, Kennedy, Holzman, \& Ehrenreich-May, 2020).

The resultant classes may also help to develop and apply transdiagnostic programs based on the common elements approach, which focuses on the common therapeutic procedures used for the disorders. To this effect, there are psychological programs that have proved effective for improving irritability. Derella, Johnston, Loeber, and Burke (2019) showed that the Stop Now and Plan program is effective for reducing irritability in boys with ODD via improved emotion regulation skills, a technique also used for depression and anxiety (Ehrenreich et al., 2009). In the same line, problem solving skills training is an effective technique commonly used for disruptive behavior disorders (Kazdin, 2018) and is also useful for depression and anxiety (Ugueto, Santucci, Krumholz, \& Weisz, 2014). Shared effective techniques could therefore be used to target the main problems in each group. While for class 3 problem solving may be directed towards impulsive responding, for class 2 it may also focus on avoiding behavior as a way of managing fear or lack of energy.

Last, the results of the present study can also inform the development of transdiagnostic treatments based on the principle guide approach, focusing on the core principles of therapeutic change in the disorders and potentially refining and adapting the programs to the specific needs of the different classes. Several cognitive behavioral approaches have already proved effective in dealing with dysregulated emotions (Perepletchikova et al., 2017; Sanders, Markie-Dadds, \& Turner, 2013; Webster-Stratton \& Reid, 2004). 
Trajectories of irritability, oppositionism, depression and anxiety 22

\section{Acknowledgement}

This work was supported by the Spanish Ministry of Science, Innovation and Universities

[Grant PGC2018-095239-B-I00 (MICIU/FEDER)] 


\section{References}

Achenbach, T. M., \& Rescorla, L. A. (2000). Manual for the ASEBA preschool-age forms \& Profiles. Burlington, VT: University of Vermont, Research Center for Children, Youth \& Families.

Achenbach, T. M., \& Rescorla, L. A. (2003). Manual for the ASEBA adult forms \& profiles. Burlington, VT: University of Vermont, Research Center for Children, Youth \& Families.

Althoff, R. R., Kuny-Slock, A. V., Verhulst, F. C., Hudziak, J. J., \& van der Ende, J. (2014). Classes of oppositional-defiant behavior: Concurrent and predictive validity. Journal of Child Psychology \& Psychiatry, 55, 1162-1171. doi: 10.1111/jcpp.12233

Angold, A., Costello, E. J., \& Erkanli, A. (1999). Comorbidity. Journal of Child Psychology and Psychiatry, 40, 57-87.

Brotman, M. A., Kircanski, K., Stringaris, A., Pine, D. S., \& Leibenluft, E. (2017). Irritability in Youths: A Translational Model. American Journal of Psychiatry, 174(6), 520-532. doi: 10.1176/appi.ajp.2016.16070839

Burke, J. D., Boylan, K., Rowe, R., Duku, E., Stepp, S. D., Hipwell, A. E., \& Waldman, I. D. (2014). Identifying the irritability dimension of odd: application of a modified bifactor model across five large community samples of children. Journal of Abnormal Psychology, 123(4), 841-851. doi: 10.1037/a0037898

Burke, J. D., Hipwell, A. E., \& Loeber, R. (2010). Dimensions of oppositional defiant disorder as predictors of depression and conduct disorder in preadolescent girls. Journal of the American Academy of Child Adolescent Psychiatry, 49, 484-492. doi: 10.1016/j.jaac.2010.01.016

Cohen, J. (1992). A power primer. Psychological Bulletin, 112 155-159. 
Chahal, R., Gotlib, I. H., \& Guyer, A. E. (2020) Research Review: Brain network connectivity and the heterogeneity of depression in adolescence - a precision mental health perspective. Journal of Child Psychology and Psychiatry. doi: 10.1111/jcpp.13250

Chu, B. C., Temkin, A. B., \& Toffey, K. (2016). Transdiagnostic mechanisms and treatment for children and adolescents: An emerging field: Oxford Handbooks Online.

de la Osa, N., Granero, R., Penelo, E., Doménech, J. M., \& Ezpeleta, L. (2014). Psychometric properties of the Alabama Parenting Questionnaire - Preschool revision (APQ-Pr) in 3 year-old Spanish preschoolers. Journal of Child and Family Studies, 23, 776-784. doi: 10.1007/s10826-013-9730-5

de Lijster, J. M., van den Dries, M. A., van der Ende, J., Utens, E. M. W. J., Jaddoe, V. W., Dieleman, G. C., . . . Legerstee, J. S. (2019). Developmental trajectories of anxiety and depression symptoms from early to middle childhood: A population-based cohort study in the netherlands. Journal of Abnormal Child Psychology, 47(11), 1785-1798. doi: 10.1007/s10802-019-00550-5

Derella, O. J., Johnston, O. G., Loeber, R., \& Burke, J. D. (2019). CBT-Enhanced Emotion Regulation as a Mechanism of Improvement for Childhood Irritability. Journal of Clinical Child and Adolescent Psychology, 48, S146-S154. doi:

10.1080/15374416.2016.1270832

Ehrenreich, J. T., Goldstein, C. R., Wright, L. R., \& Barlow, D. H. (2009). Development of a Unified Protocol for the Treatment of Emotional Disorders in Youth. Child \& Family Behavior Therapy, 31(1), 20-37. doi: 10.1080/07317100802701228

Ezpeleta, L., de la Osa, N., \& Doménech, J. M. (2014). Prevalence of DSM-IV disorders, comorbidity and impairment in 3-year-old Spanish preschoolers. Social Psychiatry and Psychiatric Epidemiology, 49(1), 145-155. doi: 10.1007/s00127-013-0683-1 
Ezpeleta, L., de la Osa, N., Granero, R., Domènech, J. M., \& Reich, W. (2011). The Diagnostic Interview of Children and Adolescents for Parents of Preschool and Young Children: psychometric properties in the general population. Psychiatry Research, 190, 137-144. doi: 10.1016/j.psychres.2011.04.034

Ezpeleta, L., Granero, R., de la Osa, N., Trepat, E., \& Domènech, J. M. (2016). Trajectories of oppositional defiant disorder irritability symptoms in preschool children. Journal of Abnormal Child Psychology, 44, 115-128. doi: 10.1007/s10802-015-9972-3

Ezpeleta, L., Penelo, P., de la Osa, N., Navarro, J. B., \& Trepat, E. (2019). Irritability and parenting practices as mediational variables between temperament and affective, anxiety and oppositional defiant problems. Aggressive Behavior, 45, 550-560. doi: 10.1002/ab.21850

Fanti, K. A., \& Henrich, C. C. (2010). Trajectories of pure and co-occurring internalizing and externalizing problems from age 2 to age 12: Findings from the National Institute of Child Health and Human Development Study of Early Child Care. Developmental Psychology, 46, 1159-1175. doi: 10.1037/a0020659

Forbes, E. E., \& Dahl, R. E. (2012). Research Review: Altered reward function in adolescent depression: what, when and how? Journal of Child Psychology and Psychiatry, 53(1), 3-15. doi: 10.1111/j.1469-7610.2011.02477.x

Frick, P. J. (1991). Alabama Parenting Questionnaire. Unpublished rating scale. University of Alabama.

Goodman, R. (1997). The Strengths and Difficulties Questionnaire: A Research Note. Journal of Child Psychology and Psychiatry, 38, 581-586 doi: 10.1111/j.14697610.1997.tb01545.x 
Goodman, R. (2001). Psychometric properties of the Strenghts and Difficulties Questionnaire. Journal of the American Academy of Child and Adolescent Psychiatry, 40, 1337-1345. doi: 10.1097/00004583-200111000-00015

Goodman, K. E., Rouse, M. H., Connell, A. M., Broth, M. R., Hall, C. M., \& Heyward, D. (2011). Maternal depression and child psychopathology: A meta-analytic review. Clinical Child and Family Psychology Review, 14, 1-27.

Hawks, J. L., Kennedy, S. M., Holzman, J. B. W., \& Ehrenreich-May, J. (2020). Development and application of an innovative transdiagnostic treatment approach for pediatric irritability. Behavior Therapy, 51(2), 334-349. doi: 10.1016/j.beth.2019.07.004

Holm, S. (1979). A simple sequentially rejective multiple test procedure. Scandinavian Journal of Statistics, 6, 65-70.

Hollingshead, A. B. (1975). Four factor index of social status. New Haven, CT: Unpublished manuscript, Yale University, Department of Sociology.

Ip, K. I., Jester, J. M., Sameroff, A., \& Olson, S. L. (2019). Linking Research Domain Criteria (RDoC) constructs to developmental psychopathology: The role of self-regulation and emotion knowledge in the development of internalizing and externalizing growth trajectories from ages 3 to 10. Development and Psychopathology, 31(4), 1557-1574. doi: $10.1017 / s 0954579418001323$

Kazdin, A. E. (2018). Implementation and evaluation of treatments for children and adolescents with conduct problems: Findings, challenges, and future directions. Psychotherapy Research, 28(1), 3-17. doi: 10.1080/10503307.2016.1208374

Krieger, F. V., Polanczyk, V. G., Robert, G., Rohde, L. A., Graeff-Martins, A. S., Salum, G., . . Stringaris, A. (2013). Dimensions of oppositionality in a Brazilian community sample: Testing the DSM-5 proposal and etiological links. Journal of the American Academy of Child and Adolescent Psychiatry, 52(4), 389-400.e381. 
Lavigne, J. V., Gouze, K. R., Bryant, F. B., \& Hopkins, J. (2014). Dimensions of oppositional defiant disorder in young children: Heterotypic continuity with anxiety and depression. . Journal of Abnormal Child Psychology, 42, 937-951. doi: 10.1007/s10802-014-9853-1

Leadbeater, B., Thompson, K., \& Gruppuso, V. (2012). Co-occurring trajectories of symptoms of anxiety, depression, and oppositional defiance from adolescence to young adulthood. Journal of Clinical Child and Adolescent Psychology, 41(6), 719730. doi: 10.1080/15374416.2012.694608

Marchette, L. K., \& Weisz, J. R. (2017). Practitioner Review: Empirical evolution of youth psychotherapy toward transdiagnostic approaches. Journal of Child Psychology and Psychiatry, 58(9), 970-984. doi: 10.1111/jcpp.12747

Matthys, W., Vanderschuren, L. J. M. J., \& Schutter, D. J. L. G. (2013). The neurobiology of oppositional defiant disorder and conduct disorder: Altered functioning in three mental domains. Development and Psychopathology, 25(1), 193-207. doi: $10.1017 / \mathrm{s} 0954579412000272$

Oh, Y., Greenberg, M. T., Willoughby, M. T., \& Family Life Project, K. (2020). Examining Longitudinal Associations between Externalizing and Internalizing Behavior Problems at Within- and Between-Child Levels. Journal of Abnormal Child Psychology, 48(4), 467-480. doi: 10.1007/s10802-019-00614-6

Oldehinkel, A. J., Verhulst, F. C., \& Ormel, J. (2011). Mental health problems during puberty: Tanner stage-related differences in specific symptoms. The TRAILS study. Journal of Adolescence, 34(1), 73-85. doi: 10.1016/j.adolescence.2010.01.010

Pagliaccio, D., Pine, D. S., Barch, D. M., Luby, J. L., \& Leibenluft, E. (2018). Irritability trajectories, cortical thickness, and clinical outcomes in a sample enriched for 
preschool depression. Journal of the American Academy of Child and Adolescent Psychiatry, 57(5), 336-+. doi: 10.1016/j.jaac.2018.02.010

Patterson, G. (2002). The early development of coercive family process. In J. Reid, G. Patterson \& J. Snyder (Eds.), Antisocial behavior in children and adolescents: a developmental analysis and the Oregon model for intervention (pp. 25-44). Washington, DC: APA.

Perepletchikova, F., Nathanson, D., Axelrod, S. R., Merrill, C., Walker, A., Grossman, M., .. . Walkup, J. (2017). Randomized Clinical Trial of Dialectical Behavior Therapy for Preadolescent Children With Disruptive Mood Dysregulation Disorder: Feasibility and Outcomes. Journal of the American Academy of Child and Adolescent Psychiatry, 56(10), 832-840. doi: 10.1016/j.jaac.2017.07.789

Putnam, S. P., \& Rothbart, M. K. (2006). Development of short and very short forms of the Children's Behavior Questionnaire Journal of Personality Assessment, 87, 103-113.

Rowe, R., Costello, E. J., Angold, A., Copeland, W. E., \& Maughan, B. (2010). Developmental pathways in oppositional defiant disorder and conduct disorder. Journal of Abnormal Psychology, 119(4), 726-738. doi: 10.1037/a0020798

Sallis, H., Szekely, E., Neumann, A., Jolicoeur-Martineau, A., van Ijzendoorn, M., Hillegers, M., .. . Evans, J. (2019). General psychopathology, internalising and externalising in children and functional outcomes in late adolescence. Journal of Child Psychology and Psychiatry, 60(11), 1183-1190. doi: 10.1111/jcpp.13067

Sanders, M. R., Markie-Dadds, C., \& Turner, K. M. T. (2013). Practitioner Manual for Standard Triple P (2nd ed.). Brisbane, Australia: Triple P International.

Sellers, R., Collishaw, S., Rice, F., Thapar, A. K., Potter, R., Mars, B., . . Thapar, A. (2013). Risk of psychopathology in adolescent offspring of mothers with psychopathology and 
recurrent depression. British Journal of Psychiatry, 202, 108-114. doi:

10.1192/bjp.bp.111.104984

Silk, J. S., Davis, S., McMakin, D. L., Dahl, R. E., \& Forbes, E. E. (2012). Why do anxious children become depressed teenagers? The role of social evaluative threat and reward processing. Psychological Medicine, 42(10), 2095-2107. doi:

$10.1017 / \mathrm{s} 0033291712000207$

Smith, J. D., Wakschlag, L., Krogh-Jespersen, S., Walkup, J. T., Wilson, M. N., Dishion, T. J., \& Shaw, D. S. (2019). Dysregulated irritability as a window on young children's psychiatric risk: transdiagnostic effects via the family check-up. Development and Psychopathology, 31(5), 1887-1899. doi: 10.1017/s0954579419000816

Stringaris, A. (2011). Irritability in children and adolescents: a challenge for DSM-5. European Child and Adolescent Psychiatry, 20(2), 61-66. doi: 10.1007/s00787-0100150-4

Stringaris, A., \& Goodman, R. (2009). Three dimensions of oppositionality in youth. Journal of Child Psychology and Psychiatry, 50(3), 216-223. doi: 10.1111/j.14697610.2008.01989.x

Stringaris, A., Vidal-Ribas, P., Brotman, M. A., \& Leibenluft, E. (2018). Practitioner Review: Definition, recognition, and treatment challenges of irritability in young people. Journal of child psychology and psychiatry, and allied disciplines, 59, 721-739. doi: 10.1111/jcpp.12823

Toohey, M. J., \& DiGiuseppe, R. (2017). Defining and measuring irritability: Construct clarification and differentiation. Clinical Psychology Review, 53, 93-108. doi: 10.1016/j.cpr.2017.01.009

Ugueto, A. M., Santucci, L. C., Krumholz, L. S., \& Weisz, J. R. (2014). Problem-solving skills training. In E. S. Sburlati, H. J. Lyneham, C. A. Schniering \& R. M. Rapee 
(Eds.), Evidence-based CBT for anxiety and depression in children and adolescents (pp. 247-259).

Vidal-Ribas, P., Brotman, M. A., Valdivieso, I., Leibenluft, E., \& Stringaris, A. (2016). The status of irritability in psychiatry: A conceptual and quantitative review. Journal of the American Academy of Child and Adolescent Psychiatry, 55, 556-570. doi: 10.1016/j.jaac.2016.04.014

Wakschlag, L. S., Estabrook, R., Petitclerc, A., Henry, D., Burns, J. L., Perlman, S. B., . . Briggs-Gowan, M. L. (2015). Clinical implications of a dimensional approach: The normal:abnormal spectrum of early irritability. Journal of the American Academy of Child and Adolescent Psychiatry, 54(8), 626-634. doi: 10.1016/j.jaac.2015.05.016

Wakschlag, L. S., Perlman, S. B., Blair, R. J., Leibenluft, E., Briggs-Gowan, M. J., \& Pine, D. S. (2018). The neurodevelopmental basis of early childhood disruptive behavior: Irritable and callous phenotypes as exemplars. American Journal of Psychiatry, 175(2), 114-130. doi: 10.1176/appi.ajp.2017.17010045

Wakschlag, L. S., Roberts, M. Y., Flynn, R. M., Smith, J. D., Krogh-Jespersen, S., Kaat, A. J., ... Davis, M. M. (2019). Future directions for early childhood prevention of mental disorders: A road map to mental health, earlier. Journal of clinical child \& adolescent psychology, 1-16. doi: 10.1080/15374416.2018.1561296

Webster-Stratton, C., \& Reid, M. J. (2004). Strengthening social and emotional competence in young children - The foundation for early school readiness and success - Incredible years classroom social skills and problem-solving curriculum. Infants and Young Children, 17(2), 96-113.

Wickrama, K. A. S., Lee, T. K., Walker-O'Neal, C., \& Lorenz, F. O. (2016). Higher-order growth curves and mixture modeling with MPlus. A pratical guide. . New York: Routledge. 
Wiggins, J. L., Mitchell, C., Stringaris, A., \& Leibenluft, E. (2014). Developmental Trajectories of Irritability and Bidirectional Associations With Maternal Depression Journal of the American Academy of Child Adolescent Psychiatry, 53, 1191-1205. doi: 10.1016/j.jaac.2014.08.005

Willner, C. J., Gatzke-Kopp, L. M., \& Bray, B. C. (2016). The dynamics of internalizing and externalizing comorbidity across the early school years. Development and Psychopathology, 28(4), 1033-1052. doi: 10.1017/s0954579416000687 
Table 1.

Fitting Indices for One to Six Class LCGAs

\begin{tabular}{|c|c|c|c|c|c|}
\hline $\begin{array}{l}\mathrm{N} . \\
\text { classes }\end{array}$ & AIC & $\mathrm{aBIC}$ & $\begin{array}{l}\text { Class: } N \\
\text { (weighted) }\end{array}$ & $\begin{array}{c}\text { Class: } \\
\text { probability* }\end{array}$ & Entropy \\
\hline 1 & 117556.763 & 117601.929 & 1: 493 & - & - \\
\hline 2 & 113501.113 & 113555.517 & $\begin{array}{l}1: 133 \\
2: 360\end{array}$ & $\begin{array}{l}1: .975 \\
2: .994\end{array}$ & .960 \\
\hline 3 & 112362.599 & 112426.242 & $\begin{array}{l}\text { 1: } 331 \\
\text { 2: } 55 \\
\text { 3: } 107\end{array}$ & $\begin{array}{l}\text { 1: .986 } \\
\text { 2: .969 } \\
\text { 3: .983 }\end{array}$ & .961 \\
\hline 4 & 111528.433 & 111601.315 & $\begin{array}{l}\text { 1: } 51 \\
\text { 2: } 246 \\
\text { 3: } 46 \\
\text { 4: } 149\end{array}$ & $\begin{array}{l}\text { 1:.987 } \\
\text { 2:.975 } \\
\text { 3:.981 } \\
\text { 4:.950 }\end{array}$ & .946 \\
\hline 5 & 110876.641 & 110958.761 & $\begin{array}{l}\text { 1: } 190 \\
\text { 2: } 62 \\
\text { 3: } 43 \\
\text { 4: } 29 \\
\text { 5: } 167\end{array}$ & $\begin{array}{l}\text { 1:.960 } \\
\text { 2: .969 } \\
\text { 3: .979 } \\
\text { 4: .990 } \\
\text { 5: .946 }\end{array}$ & .936 \\
\hline 6 & 110503.047 & 110594.406 & $\begin{array}{l}\text { 1: } 180 \\
\text { 2: } 39 \\
\text { 3: } 10 \\
\text { 4: } 57 \\
\text { 5: } 117 \\
\text { 6: } 89\end{array}$ & $\begin{array}{l}\text { 1:.968 } \\
\text { 2:.985 } \\
\text { 3:.995 } \\
\text { 4:.973 } \\
\text { 5:.904 } \\
\text { 6: } 932\end{array}$ & .929 \\
\hline
\end{tabular}

Note. aBIC: Sample-Size Adjusted BIC.

*On-diagonal values for posterior probability of class membership. In bold: selected solution of LCGA 
Table 2

Descriptives of the Classes

\begin{tabular}{|c|c|c|c|c|c|c|c|}
\hline & \multirow{2}{*}{$\begin{array}{l}\text { Class } 1 \\
n=332\end{array}$} & \multirow{2}{*}{$\begin{array}{l}\text { Class2 } \\
n=55\end{array}$} & \multirow{2}{*}{$\begin{array}{l}\text { Class } 3 \\
n=108\end{array}$} & \multicolumn{4}{|c|}{ Class comparisons } \\
\hline & & & & $p$ & 2 vs. 1 & 3 vs. 1 & 3 vs. 2 \\
\hline Sex (\% boys) & 51.7 & 52.7 & 47.2 & .704 & & & \\
\hline \multicolumn{8}{|l|}{ Socioeconomic status (\%) } \\
\hline High & 37.7 & 25.5 & 41.7 & .262 & & & \\
\hline Medium/medium-high & 46.4 & 47.3 & 43.5 & & & & \\
\hline Medium-low/Low & 15.9 & 27.3 & 14.8 & & & & \\
\hline \multicolumn{8}{|l|}{ Variables at baseline age 3} \\
\hline$($ mean $\mathrm{T})$ & & & & & & & \\
\hline Irritability & 47.62 & 56.45 & 53.91 & $<.001$ & $<.001$ & $<.001$ & .161 \\
\hline Oppositional problems & 46.90 & 55.48 & 56.19 & $<.001$ & $<.001$ & $<.001$ & .684 \\
\hline Depression problems & 47.55 & 60.04 & 48.96 & $<.001$ & $<.001$ & .157 & $<.001$ \\
\hline Anxiety problems & 47.87 & 59.41 & 49.74 & $<.001$ & $<.001$ & .083 & $<.001$ \\
\hline
\end{tabular}

Class1: Low; Class2: High Depression-Anxiety-Irritability-plus ODD; Class3: Medium-high ODD-Irritability In bold: significant differences 
Table 3.

Baseline and Outcomes Comparison between the 3 Classes

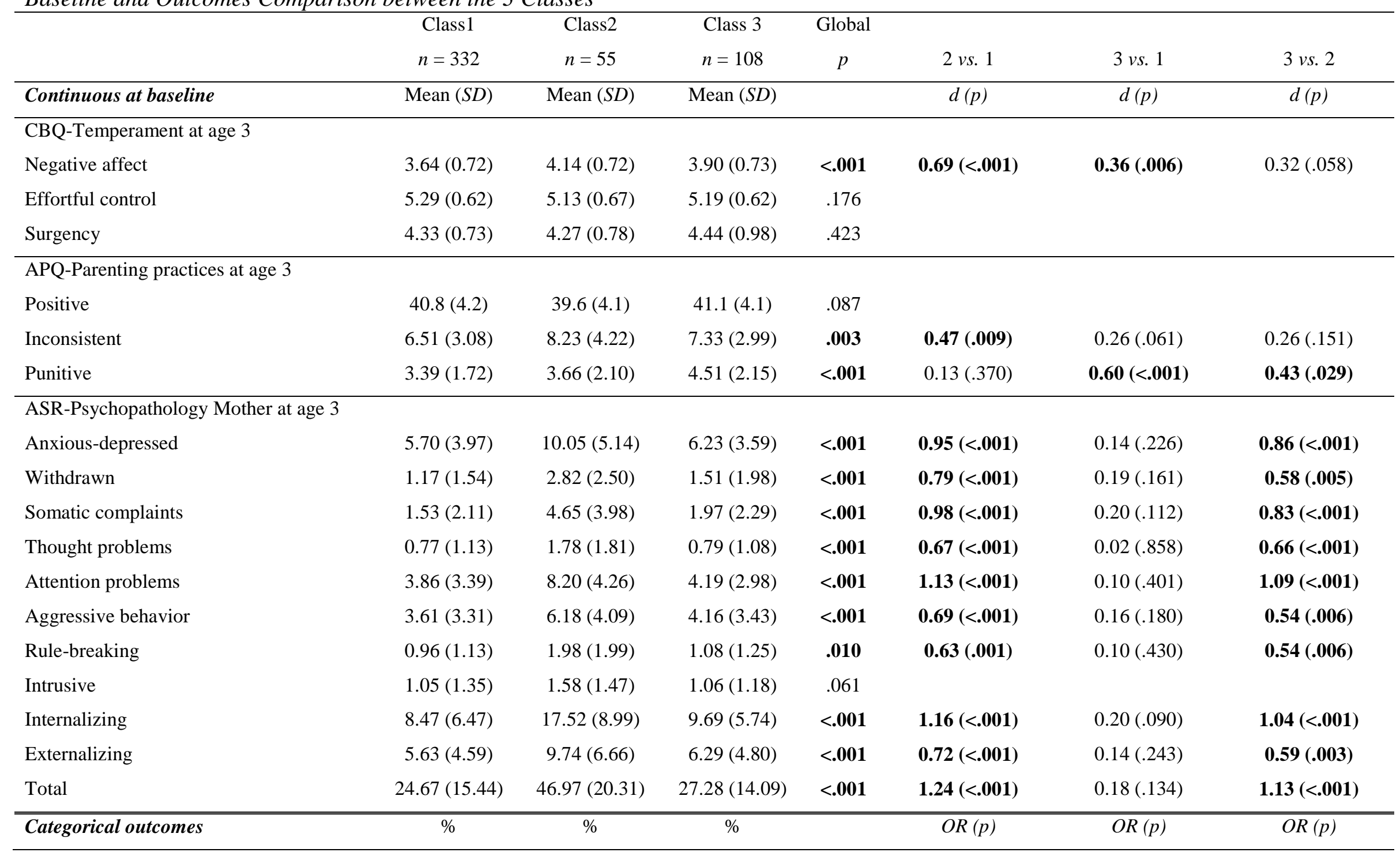




\begin{tabular}{|c|c|c|c|c|c|c|c|}
\hline \multicolumn{8}{|l|}{ DSM-5 diagnoses at age 11} \\
\hline Attention-deficit/hyperactivity disorder & 4.7 & 25.5 & 8.0 & $<.001$ & $5.66(<.001)$ & $1.62(.380)$ & $0.29(.058)$ \\
\hline Oppositional defiant disorder & 2.7 & 24.0 & 24.1 & $<.001$ & $10.22(<.001)$ & $10.93(<.001)$ & $1.07(.881)$ \\
\hline Any anxiety & 6.3 & 14.5 & 3.7 & .025 & $5.88(<.001)$ & $4.20(.001)$ & $0.71(.468)$ \\
\hline Professional consultation at age 12 & 15.6 & 53.3 & 34.5 & $<.001$ & $6.06(<.001)$ & $2.87(.002)$ & $0.47(.059)$ \\
\hline Treatment for any disorder at age 12 & 3.8 & 24.4 & 15.5 & $<.001$ & $8.45(<.001)$ & $4.96(.004)$ & $0.59(0.278)$ \\
\hline Continuous outcomes & Mean $(S D)$ & Mean $(S D)$ & Mean $(S D)$ & & $d(p)$ & $d(p)$ & $d(p)$ \\
\hline Comorbidity at age 11 & $0.29(0.58)$ & $1.45(1.72)$ & $0.79(0.86)$ & $<.001$ & $0.90(<.001)$ & $0.68(<.001)$ & $0.48(<.001)$ \\
\hline \multicolumn{8}{|l|}{ SDQ-Parents at age 12} \\
\hline Emotion & $0.65(1.10)$ & $2.05(1.72)$ & $1.83(1.56)$ & $<.001$ & $0.97(<.001)$ & $0.87(<.001)$ & $0.14(.864)$ \\
\hline Conduct & $0.53(0.84)$ & $1.49(1.55)$ & $1.65(1.26)$ & $<.001$ & $0.77(.005)$ & $1.04(<.001)$ & $0.11(.478)$ \\
\hline Hyperactivity & $1.89(2.12)$ & $4.07(2.68)$ & $3.29(2.13)$ & $<.001$ & $0.90(.003)$ & $0.66(<.001)$ & $0.32(.575)$ \\
\hline Peer & $0.50(1.02)$ & $1.65(1.92)$ & $1.12(1.42)$ & .001 & $0.75(.023)$ & $0.50(.012)$ & $0.31(.349)$ \\
\hline Prosocial & $1.13(1.34)$ & $1.82(1.72)$ & $1.59(1.45)$ & .007 & $0.45(.128)$ & $0.33(.115)$ & $0.15(.658)$ \\
\hline Total & $3.56(3.44)$ & $9.26(5.09)$ & 7.89 (3.63) & $<.001$ & $1.31(<.001)$ & $1.22(<.001)$ & $0.31(.709)$ \\
\hline \multicolumn{8}{|l|}{ SDQ-Teachers at age 12} \\
\hline Emotion & 1.12 (1.69) & $2.97(2.46)$ & $1.69(1.90)$ & $<.001$ & $0.88(<.001)$ & $0.32(.049)$ & $0.58(.024)$ \\
\hline Conduct & $0.88(1.41)$ & $1.61(1.85)$ & $1.32(1.76)$ & $<.001$ & $0.44(.081)$ & $0.28(.379)$ & $0.16(.323)$ \\
\hline Hyperactivity & $2.21(2.60)$ & 3.75 (3.18) & $2.94(2.80)$ & $<.001$ & $0.53(.057)$ & $0.27(.260)$ & $0.27(.272)$ \\
\hline Peer & $1.04(1.56)$ & $2.30(1.82)$ & $1.41(1.98)$ & .001 & $0.73(<.001)$ & $0.20(.229)$ & $0.47(.066)$ \\
\hline Prosocial & $2.12(2.21)$ & $3.22(2.30)$ & $2.57(2.14)$ & .007 & $0.48(.036)$ & $0.21(.218)$ & $0.29(.330)$ \\
\hline Total & $5.26(5.19)$ & $10.62(6.46)$ & $7.37(6.12)$ & $<.001$ & $0.91(<.001)$ & $0.37(.043)$ & $0.52(.039)$ \\
\hline Functional impairment at age 12 (CGAS) & $74.16(7.91)$ & $64.02(11.55)$ & $65.18(8.85)$ & $<.001$ & $1.02(<.001)$ & $1.07(<.001)$ & $0.11(.828)$ \\
\hline
\end{tabular}


Trajectories of irritability, oppositionism, depression and anxiety 36
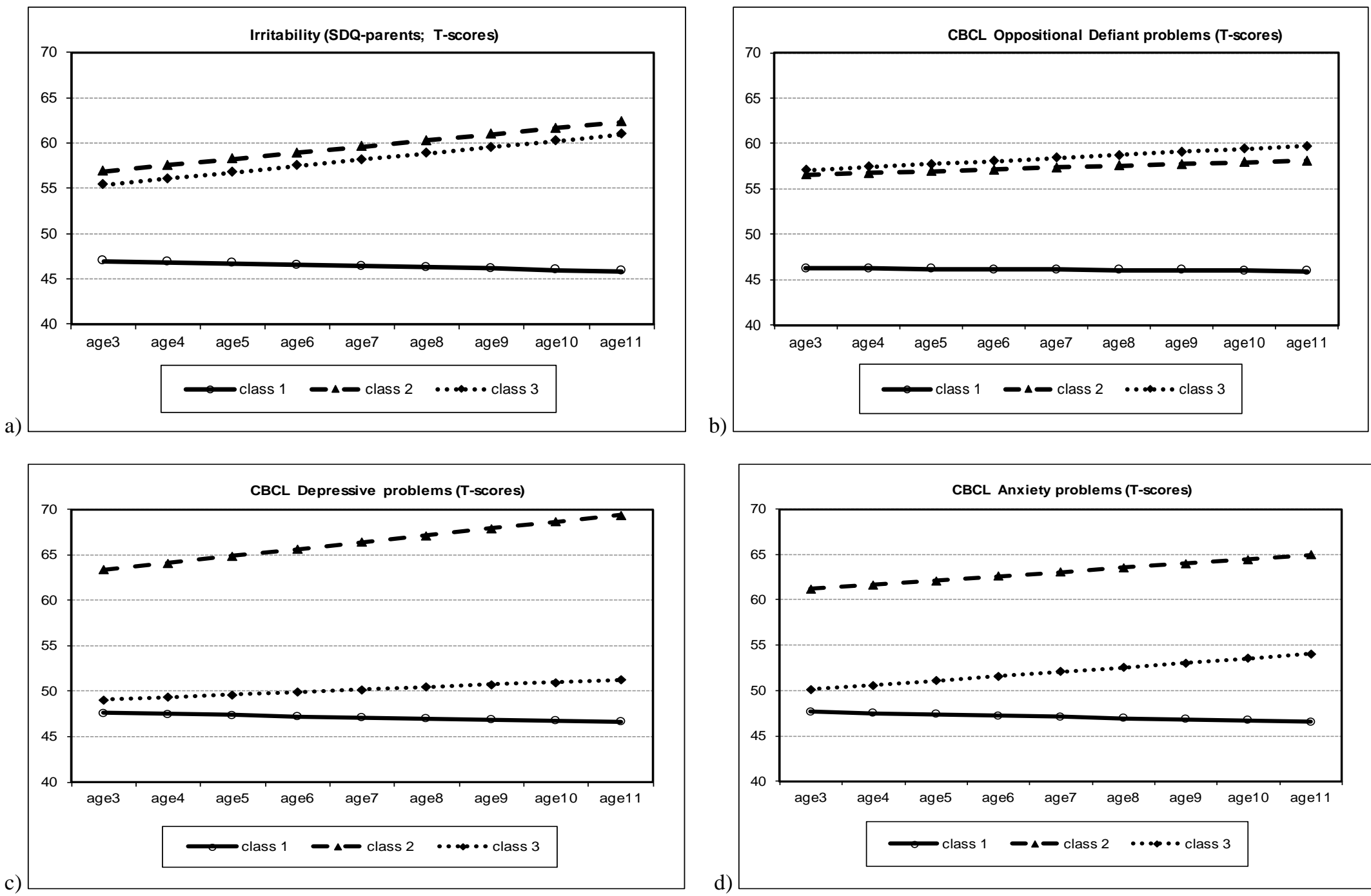

Figure 1. Trajectories for SDQ-Irritability and CBCL scores by classes ( $N$ weighted) separately for each measure. 
Trajectories of irritability, oppositionism, depression and anxiety 37
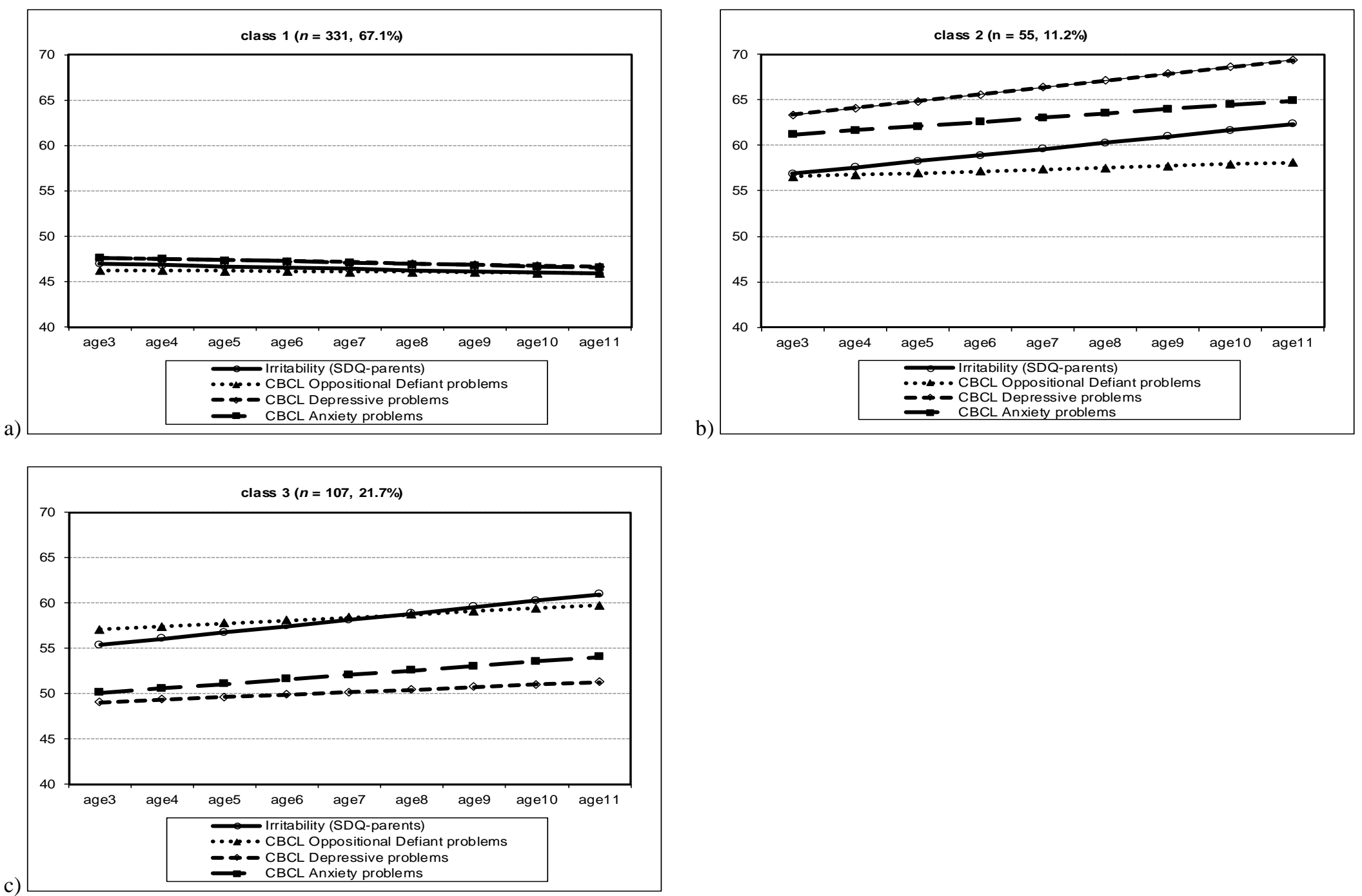

Figure 2. Trajectories for SDQ-Irritability and CBCL scores by classes ( $N$ weighted) for each resultant class combining all four measures. 